\title{
VIRTUAL ENDOSCOPY : FROM SIMULATION TO OPTIMIZATION OF AN ACTIVE ENDOSCOPE
}

\author{
Christofer KühL ${ }^{1}$ And Georges Dumont ${ }^{2}$
}

\begin{abstract}
The design and realization of micro robotic devices in the medical field enable the operating gesture to be assisted. So a polyarticulated device actuated with shape memory alloy (SMA) springs for endoscopy is developed in collaboration with the Laboratoire de Robotique de Paris. In this paper, a simulator realization, based on an original simulation platform (OpenMASK), of this structure is described. A numerical representation of the inspected network obtained by magnetic resonance imaging is used. A contact detection algorithm, which will be described, allows to determine the interaction forces between the endoscope and the duct thanks to a distance cartography of the space, in order to minimize the calculation time. An important experimental work shall be lead to determine the parameters of the contact model. Simulation results of virtual navigation through this duct representation by using an endoscope mechanical model including a behaviour description of the SMA actuators will be presented. Moreover, the efficiency of a command with the multi-agent approach is proved. Then the simulator interest is studied for the prototype amelioration by applying the techniques of virtual prototyping. Some results of optimization by using genetic algorithms with respect to the virtual navigation task will be shown.
\end{abstract}

Résumé. La conception et la réalisation de systèmes micro robotiques dans le domaine médical permettent une aide au geste opératoire. C'est pourquoi nous développons en collaboration avec le Laboratoire de Robotique de Paris un système polyarticulé actionné par des ressorts en alliage à mémoire de forme (AMF) destiné à l'endoscopie. Dans cet article, nous décrivons la réalisation d'un simulateur de cette structure s'appuyant sur une plate-forme originale de simulation (OpenMASK). Nous utilisons une représentation numérique, construite à partir d'imagerie à résonance magnétique, du réseau à inspecter. L'algorithme de détection du contact qui sera décrit nous permet de déterminer les efforts d'interaction entre l'endoscope et le conduit à partir d'une cartographie des distances préétablie afin de minimiser le temps de calcul. Un important travail expérimental doit être mené afin de déterminer les paramètres des efforts d'interaction. Nous présenterons des résultats de simulation de la navigation virtuelle à travers ce réseau d'un modèle mécanique d'endoscope incluant une description du comportement des actionneurs en AMF. Le simulateur nous a par ailleurs permis de montrer l'efficacité d'une commande par l'approche multi-agents. Nous étudions ensuite l'intérêt de ce simulateur pour l'amélioration du prototype en appliquant les techniques du prototypage virtuel. Nous montrerons la faisabilité de la méthode avec des résultats d'optimisation utilisant des algorithmes génétiques.

\footnotetext{
1 ENS Cachan - Antenne de Bretagne, Campus de Ker Lann, 35170 Bruz, 02-99-05-52-71,

Christofer.Kuhl@bretagne.ens-cachan.fr

2 IRISA, campus de beaulieu, 35042 Rennes Cédex, 02-99-84-25-74, et ENS Cachan, Antenne de Bretagne,

Georges.Dumont@bretagne.ens-cachan.fr
} 


\section{INTRODUCTION}

In the medical field, a strong demand is expressed by the surgeons to realize less invasive inspection and operation devices. An answer to this problem is given by the micro robotics systems which will enable the operating gesture to be assisted thanks to active endoscopes realization. The use of such systems can be spread into wide fields of applications as for example the inspection of ducts in the nuclear field. The CNRS demonstrator "micro inspect intra tube" demand gave us the opportunity to develop a collaboration between various organisms :

- The LRP (Laboratoire de Robotique de Paris) for the conception of an endoscope with distributed SMA (Shape Memory Alloy) actuators ;

- The ENS (Ecole Normale Supérieure de Cachan - Antenne de Bretagne) and the IRISA (Institut de Recherche en Informatique et Systèmes Aléatoires) for the development of a simulator aimed at optimizing the developed prototypes.

\section{Endoscopes}

There are several kinds of endoscopes : their application domain, their size and their morphology distinguish them. The flexible endoscopes (See figure 1) are devices provided with optical fibres which convey the image up to an ocular. The ocular allows a direct vision or an adaptation of a camera. Their diameter varies from 5 to $15 \mathrm{~mm}$. The device head is generally directional through an orientation system. This one is constituted by four cables connected to the head of the endoscope and which are pulled by the surgeon by means of knurls.
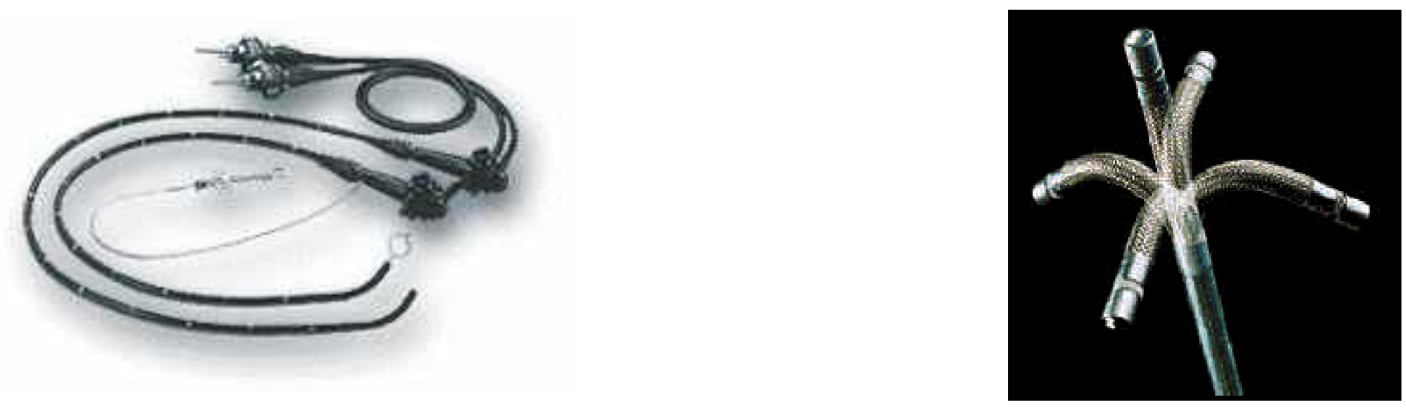

FiguRE 1. Endoscope with orientation system

A new generation of active endoscopes was developed by Olympus [1]. The head orientation is controlled through flexion modules activated by SMA. However, these systems do not allow to adapt their curvature to the investigated environments. Developments of new prototypes, aiming to improve the navigation of endoscopes in the inspected ducts, are in progress. A prototype developed in Japan [2] is established by trays interconnected by SMA threads (figure 2). The orientation between two trays connected by three SMA threads is controlled by piloting the length of the above-mentioned thread. The prototype developed in the LRP [3] is a stiff polyarticulated structure (figure 3). The endoscope body has an outside diameter of $8 \mathrm{~mm}$ and its length is, due to its structure, indefinite. The mechanism structure consists of a succession of modules articulated to each other by pin joints. These connections are alternatively oriented at $90^{\circ}$ to allow a $3 \mathrm{D}$ motion of the structure. The endoscope head (which will not be described here) should contain a device allowing to obtain a multi-directional vision of the observed space, this could be achieved by using a prism actuated by a polymer gel. This system is usually protected by a metallic sheath in industrial endoscopes and/or by a flexible polymer sheath in medical endoscopes. On every link, two SMA springs are mounted in an antagonist configuration to change the relative orientation. The command is provided through an integrated circuit controlling the electrical power supplied to the SMA. 


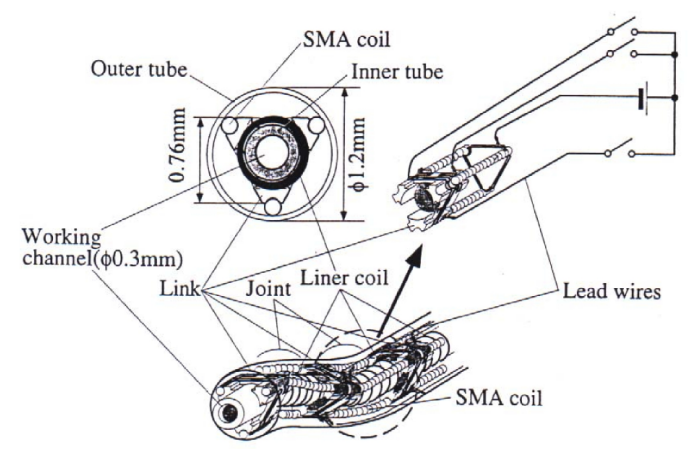

Figure 2. Multilink active catheter

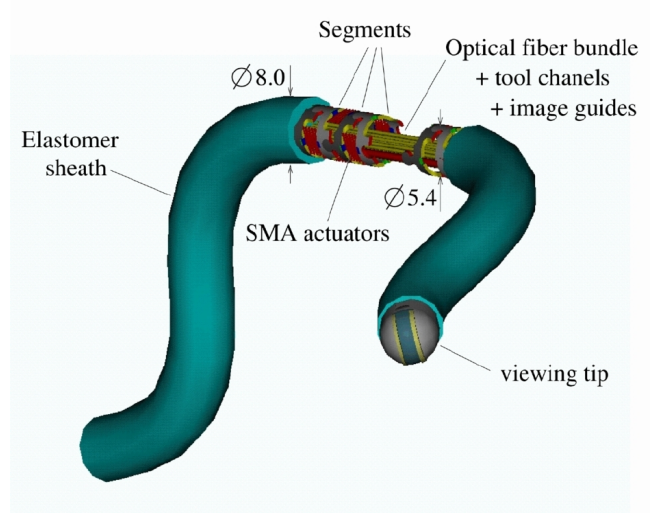

Figure 3. Polyarticulated endoscope

\section{STUdy SIMUlatoR}

The following study approaches the optimization of the conception through the development of a dedicated simulator.

\subsection{Interests of the simulator}

The simulator objective is to be able to navigate virtually in a patient body, from the reconstruction of the digestive tract obtained by a MRI. By using the techniques of virtual reality, the feeling of dumping will be maximal by using graphic and haptic interfaces. The interest of the simulator is triple :

- Young surgeons training : the training on simulator is obviously more accessible, less expensive and less risky ;

- Preoperative training : the simulator will allow the surgeon to repeat virtually the task to be made on the patient to operate ;

- Virtual prototyping [4] : the simulator allows to test the tool to estimate its quality for a given task. So we try afterward to determine, by optimization techniques based on genetic algorithms, which is the best candidate to achieve this task, so as to propose an outstanding endoscope to the surgeon.

The mechanical simulation model used and the organization in an original platform of simulation are developed here. Then the interaction model between the endoscope and the inspected channel is presented. Then the control module of SMA actuators with various reserved command strategies are described. Some interaction 
results between an artery model, directly obtained from a medical acquisition, and an endoscope model are outlined.

\subsection{OpenMASK platform}

The management of the simulator is made on the platform OpenMASK [5]. It is integrated in the SIAMES project at IRISA. The main objective of OpenMASK is to propose a modular simulation to be executed on various hardware configurations. The platform manages the synchronization and the exchanges of data between the co-operative processes even if the frequencies of calculation are different, insuring so a "dilated real time". It is developed by using the specificities of the object-oriented programming (figure 4).

In the following, the various modules of the simulator will be described.

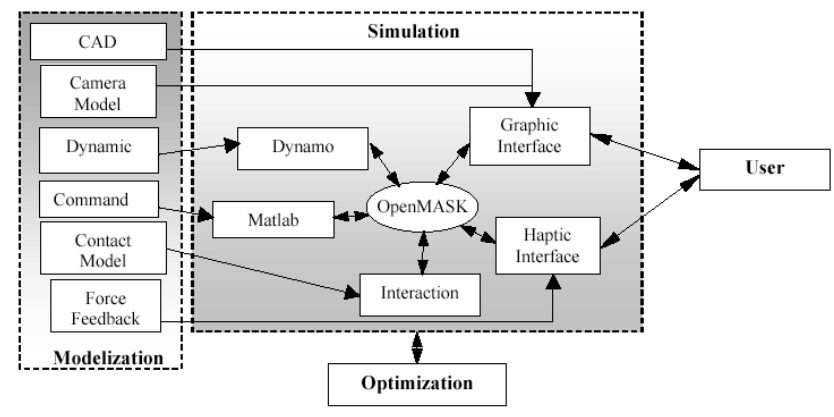

FiguRE 4. MASK : Multi-threaded Animation and Simulation Kernel

\subsection{Dynamic module}

The dynamic equations are resolved by $\mathrm{C}++$ libraries [6]. The constraints method is used. Each body keeps its six DOF. The connections are translated into term of geometrical constraints. The integrator calculates the efforts developed in the connections not to violate the geometrical constraints.

\subsection{Interaction module}

Various models $[7,8]$ are proposed to describe the behaviour of human organs or tissues, including non-linear behaviour, relaxation...

For our purpose, the objective is to develop a robust and rapid method to determine the interaction force. The reaction calculation is insured by a compliance method, because the interaction is made between a stiff body (the segments of the endoscope on one hand) and a soft body (human tissues on the other hand). The first stage of the reaction force calculation consists in collision detection between the endoscope and the channel. The detection is simply made in a geometrical way in the simulator : the surface of segments is represented by interaction points and we determine if there is any collision for each point. In that case, the following effort is applied :

$$
\vec{F}=-k \cdot \operatorname{dist} \cdot \vec{n}-f \cdot(\vec{v} \cdot \vec{n}) \cdot \vec{n}
$$

where dist is the penetration depth and $\vec{n}$ the normal orientation.

An experimental protocol will be defined to identify the parameters of the compliance model. The channel is defined by a set of facets. To detect a collision, the distances of an interaction point to each facet are calculated and the lowest one is the distance from the point to the tube. This calculation consumes obviously a lot of time. So we have developed a pre-processing method to build distance cartography in the space. The figure 5 represents the distances calculated by interpolation from the data stored for a slice of the tube. 


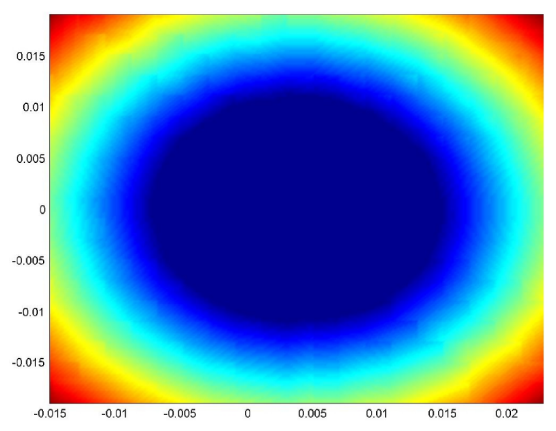

Figure 5. Distance cartography-slice

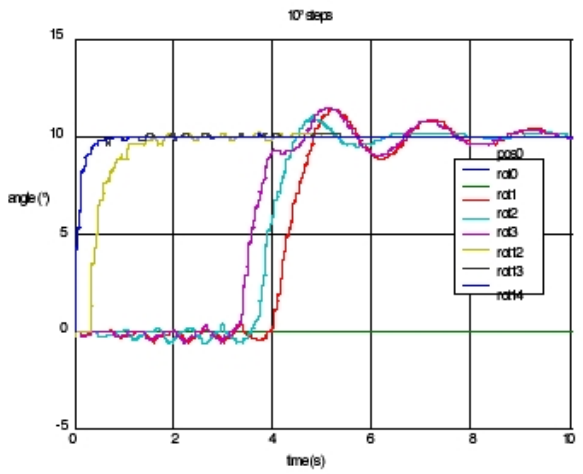

Figure 6. Response time

\subsection{Endoscope command module}

A module defining a controller which commands the mechanical model has been developed. The objective is to pilot the orientation of the endoscope segments to allow an easier introduction in the virtual duct. It is based on the geometrical description of the connections between two neighbouring segments as well as on the geometrical and behavioural characteristics of the SMA actuators [9]. On an example of an endoscope constituted by 15 segments for which we affect orders of $10^{\circ}$ orientation, we show that we have a fast system (response time of $0.4 \mathrm{~s}$ ) and very precise because the orders are finally reached (figure 6).

As the objective is to adapt the shape of the endoscope to the geometry of inspected ducts, two different strategies are proposed and analysed in the following :

- Trajectory follow-up :

The operator, thanks to the image supplied by the display system, can determine the trajectory to follow. From this trajectory (which we keep regularly passage points), we determine step by step the orders to look to each of the joints to approach in best the trajectory (figure 7 ). This strategy allows the endoscope to progress into the duct without having any contact (figure 8). This method inconvenience is that it creates an irregular movement. We are now working on a method based on genetic algorithms (on which we will come back later in this paper) to improve the endoscope progression with a smoother movement.

However, this method is valid only if the inspected channel has a constant geometry. In the case of a surgical inspection, the way through the ducts is modified in position and shape during time (breath 


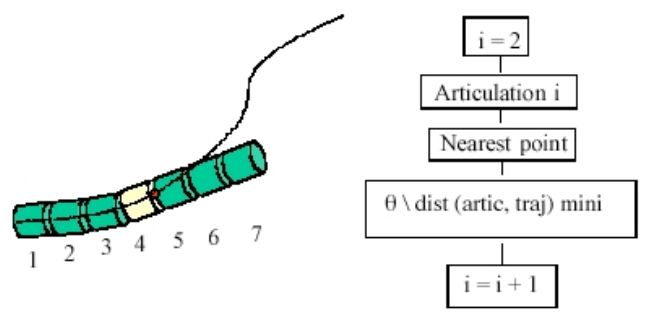

FiguRE 7. Trajectory follow-up

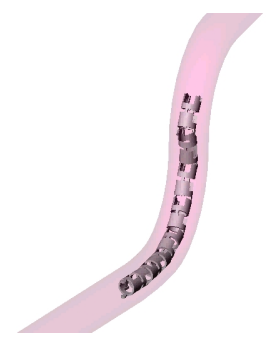

FIGURE 8. Simulation results

of the patient, heartbeat...). So the defined trajectory is not valid anymore. Then it is important to find another progress method.

- Multi-agent approach :

The objective of this approach is to have an automatic conformation of the endoscope by contact detection [10]. The solution we use relies on the split of the steering mechanism into independent subsystems and by considering them as agents. This is a very simple and modular solution independent of the length of the structure. Moreover, it is a strictly distributed approach minimizing the quantity of information exchanged between the agents. The figure 9 shows the answer of a structure constituted of two segments agents. When an effort is sensed, an order is affected to the previous joint so as to decrease the interaction effort, the opposite order is affected to the next joint approval in a way that the continuation of the endoscope becomes an unchanged orientation. In three dimensions, the agents consist of four joints because of the alternated orientation of the pin joints. So, a sensed effort activates an order distributed on both previous joints according to its orientation, and the opposite orders set on the following joints. The figure 10 shows an endoscope piloted with this strategy.

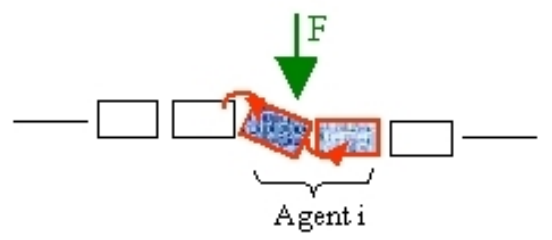

FIGURE 9. Multi-agent approach (2D principle)

This method is particularly well adapted in inspections of human networks and allows, even if there are contact zones, to strongly limit the importance of the interaction efforts. 


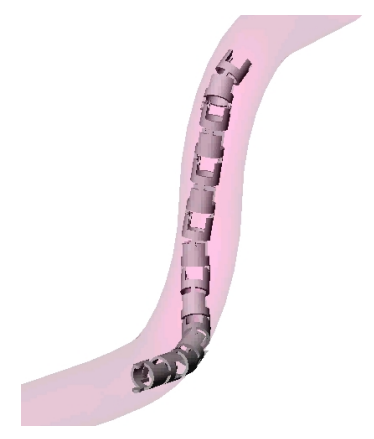

Figure 10. Simulation result

\subsection{Insertion in a medical database}

From a patient MRI, a voxelised file describing the network shape is obtained. A supplementary computer processing is made to reconstruct an image of the inspected duct. The figure 11 represents an endoscope advancing in an environment recreated from a medical database.

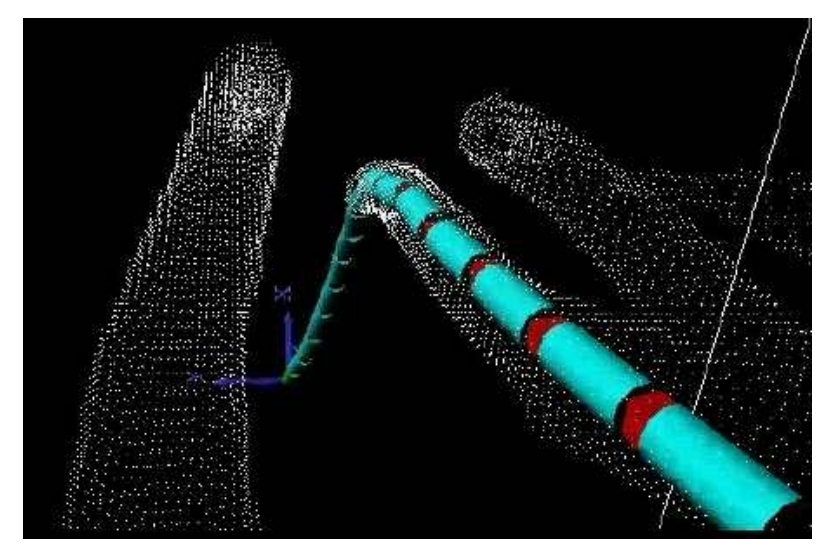

FIGURE 11. Insertion in a medical database

\subsection{Conclusion}

We developed a simulator based on a complete mechanical description of a polyarticuled active endoscope interacting with a network rebuilt from a medical data base. We also highlighted the feasibility of various command strategies (trajectory follow-up and multi-agent approach) and their limits. The first simulation results are relatively encouraging as for the speed of the simulator : at a simulation frequency of $1 \mathrm{kHz}$ for an endoscope of 15 articulations, we obtained, with a Ultra10 station with a processor Ultra SparcI at $400 \mathrm{MHz}$ and 512 Mo of RAM, a simulation time 30 times slower than the real time, which could be strongly improved by using a more powerful machine and by optimizing the computer code. A significant work must be undertaken to define a graphic interface giving a dumping feeling to the user, as well as an haptic interface to increase the handling feeling of a real endoscope.

\section{ViRTUAL PROTOTYPing}

Virtual prototyping objective is to be able to test virtually the product functionalities [11]. The designed model is tested virtually (thus the simulator must model as near as possible the mechanical behaviour of 
the structure). Its quality is measured by defining an objective function and an automatic protocol will be defined aiming at seeking the best structure for a given task. The final goal is to limit the number of real prototypes because they are obviously much more expensive than virtual prototypes. We chose to carry out this optimization work by using genetic algorithms [12]. Evolutionary algorithms allow to have a good compromise between exploration and exploitation. However, this method is admitted to be global [13].

\subsection{Methodology}

The major problem in optimization lies in the calculation time : we saw indeed that the virtual test of an endoscope was relatively long and the optimization procedure involves a great number of endoscopes tests. Thus we decided to cut out the optimization procedure in three steps :

- Purely geometrical optimization : this first stage allows to determine the articulations lengths to have a good conformation to the trajectory ;

- Degraded mechanical optimization : the trajectory follow-up is performed without using the interaction module because it is very expensive in calculation time. This stage allows a predetermination of the actuators parameters ;

- Complete mechanical optimization : the complete simulator is performed to determine the best endoscope by initializing optimization with the lengths and the mechanical parameters given by the two preceding stages.

We will develop only the first stage, the two following stages constituting our current works.

\subsection{Geometrical optimization}

Genetic algorithms copy the natural process of species evolution : an individual survives if it can adapt to the surrounding environment [14]. When it reproduces, it transmits its genes to its descent. Among these genes, some mutates naturally (figure 12).

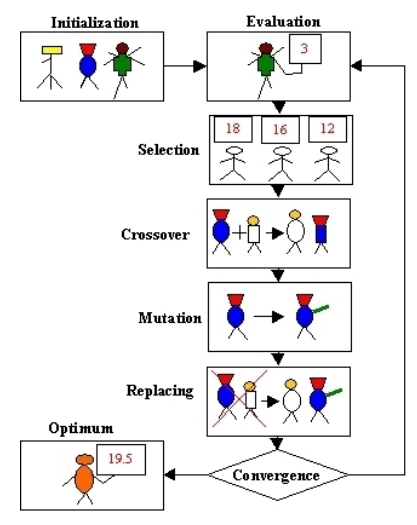

FIGURE 12. Genetic algorithms

An objective function based on the orders determination by the trajectory follow-up method is defined. The objective for the endoscope is to reach a position related to the given task. During its progress, the endoscope should interact at least with the patient tissues, i.e. to conform its shape to the trajectory defined by the average line of the channel to inspect. Thus the objective function is defined by :

$$
f=\operatorname{good} \cdot i \cdot \exp ^{-k \cdot e c a r t}
$$

where $i$ represents the index of the highest trajectory point reached by the endoscope head. It is saturated with the point index corresponding to the desired position, ecart corresponds to the cumulated gaps from endoscope 


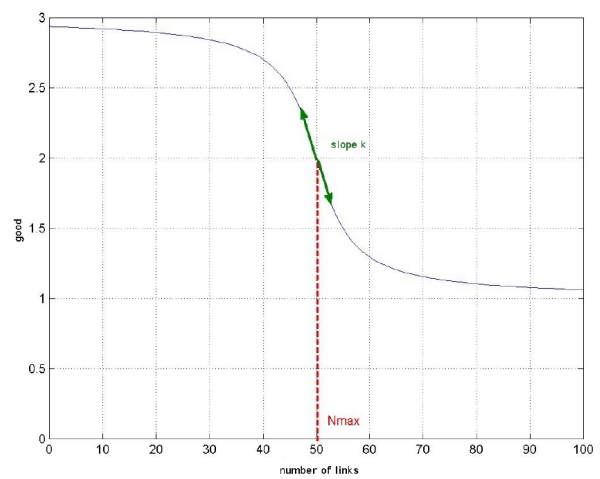

FiguRE 13. Improvement function

to trajectory during progress and good is a function (figure 13) increasing the endoscope quality when it has a small number of segments. Its goal is to decrease the complexity of the structure :

$$
\operatorname{good}=\frac{2}{\pi} \cdot\left(\pi-\arctan \left(k \cdot\left(n_{\text {artic }}-n_{\max }\right)\right)\right)
$$

\subsection{Results}

A library of genetic algorithms [15] is used. The convergence criterion of the optimization process is the number of generations. In our example, each generation consists of 40 individuals, and we carry out calculation on 80 generations. The calculation time for this example, using a Pentium III at $800 \mathrm{MHz}$ and $256 \mathrm{Mo}$ of RAM, is about 3 minutes. The optimal solution is a 31 segments endoscope. Its maximum distance with the trajectory is of $5 \mathrm{~mm}$ (figure 14). This optimization stage gives satisfactory results : indeed, the procedure doesn't find the trivial solution which is the endoscope made up of the segments of the smallest size (the solution which is the closest to the trajectory but which results in having a great number of segments).

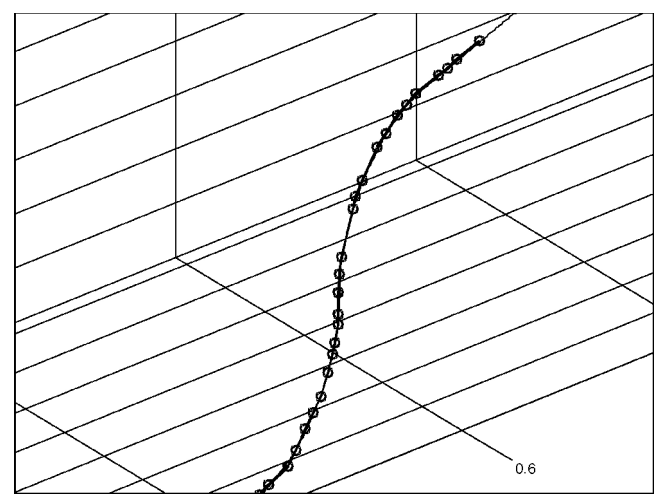

FIGURE 14. Optimized penetration depth

\subsection{Future works in virtual prototyping}

Geometrical optimization gives cheering results : it allows to predetermine endoscope segments lengths. The second stage of degraded mechanical optimization should allow to identify mechanical parameters adapted for 
the actuators choice. The optimization procedure will be very close to geometrical optimization. Initialization will be done with the last generation of geometrical optimization. Springs and damps will be added to the already calculated lengths. They will be evaluated with criteria taking into account displacements, eigen frequencies and damping in order to obtain satisfactory within the minimum of generation steps, since here time of test for each individual will be considerably increased. As far as the environment is used in the third optimization step, supplementary conditions will be introduced such as a limitation of the penetration depth of the endoscope in the duct at a not injurious distance for the patient.

\section{CONCLUSiON}

We have developed a tool allowing to simulate the progress of a polyarticulated endoscope actuated by SMA springs. This simulator is based on a mechanical description of the structure behaviour and its interacting environment, specific to the considered patient through a medical acquisition. However, work remains to be carried out in the field of interfacing. Then we showed the simulator interest in order to improve the quality of the considered prototypes. We carry out different optimization procedures by genetic algorithms in order to determine gradually the geometrical then mechanical parameters of the endoscope. When these procedures will be entirely controlled, they will be generalized to the whole system parameters (segments diameter, thrusts value) in order to be able to propose to the surgeon the most powerful structure.

\section{REFERENCES}

[1] Olympus. Apparatus for bending an insertion section of an endoscope using a SMA. United States Patent, Patent Number 4,930,494, 1990.

[2] K. Park and M. Esashi. An active catheter with integrated circuit for communication and control. In Technical Digest of the Twelfth IEEE Int. Conf. On Micro Electro Mechanical Systems (MEMS'99), pages 400-405. IEEE, January 1999.

[3] J. Szewczyk, V. De Sars, P. Bidaud, and G. Dumont. An active tubular polyarticulated micro-system for flexible endoscope. In proceedings of ISER2000 (7th International Symposium on Experimental Robotics), Hawaii, December 2000. 10-13 december 2000.

[4] G. Dumont, F. Chapelle, and P. Bidaud. Toward virtual prototyping of active endoscopes. In Proceedings of ISR2001 (International Symposium on Robotics), pages 821-826, Seoul, Korea, April 2001. IFR (International Federation of Robotics). 19-21 april 2001.

[5] S. Donikian, A. Chauffaut, T. Duval, and R. Kulpa. Gasp: from modular programming to distributed execution. In Computer Animation'98. IEEE, June 1998.

[6] B. Barenburg. Designing a class library for interactive simulation of rigid bodies. PhD thesis, Eindhoven University, 2000.

[7] Y. C. Fung. Biomechanics, Mechanical Properties of Living Tissues. Springer Verlag, 1984.

[8] K. Ikuta, K. Iritani, and J. Fukuyama. Mobile virtual endoscope system with haptic and visual information for non-invasive inspection training. In Proceedings of the 2001 ICRA (International conference on robotics and automation), pages 2037-2044, Seoul, Korea, 2001. IEEE.

[9] N. Troisfontaine and P. Bidaud. Position and force control of SMA micro-actuators. In International advanced Robotics programm, pages 110-126, October 1998.

[10] D. Duhaut. Using a multi-agent approach to solve the inverse kinematics. In Proceedings of the IROS 1993 (Intelligent Robot and System Conference), pages 2002-2007. IROS, 1993.

[11] P. Fuchs and F. Nashashibi. De la CAO à la réalité virtuelle. Revue de CFAO et d'informatique graphique, 13(2):131-167, 1998.

[12] D. E. Goldberg. Genetic algorithm in search, optimization and machine learning. Addison-Wesley pub. co, Hardcover, 1989.

[13] O. Chocron. Conception Évolutionnaire de Systèmes Robotiques. PhD thesis, Université de Paris 6, 2000.

[14] C. Darwin. The origin of species. 1859.

[15] M. Wall. Galib : a C++ library of genetic algorithm components. Technical report, Massachusetts Institute of Technology, Mechanical Engineering Department, 1996. 